\title{
Dracorhodin perchlorate induces the apoptosis of glioma cells
}

\author{
XIN CHEN*, JUNJIE LUO*, LINGHU MENG, TAIFENG PAN, BINJIE ZHAO, \\ ZHEN-GANG TANG and YONGJIAN DAI
}

\begin{abstract}
Department of Neurosurgery, Renmin Hospital, Hubei University of Medicine, Shiyan, Hubei 442000, P.R. China
\end{abstract}
Received November 9, 2015; Accepted December 17, 2015

DOI: $10.3892 /$ or.2016.4612

\begin{abstract}
Dracorhodin perchlorate (Dp), a synthetic analogue of the antimicrobial anthocyanin red pigment, has recently been shown to induce apoptotic cell death in various types of cancer cells. Yet, the inhibitory effect of Dp on human glioma cells remains uninvestigated. Therefore, in the present study, 3-[4,5-dimethylthiazol-2-yl]-2,5-diphenyltetrazolium bromide (MTT) assay and flow cytometry were used to detect cell viability and cell cycle progression in glioma U87MG and T98G cells, respectively. Annexin V-FITC/propidium iodide double staining and JC-1 staining were separately applied to determine cellular apoptosis and mitochondrial membrane potential damage in the cells. The expression levels of associated proteins involved in cell cycle progression and apoptosis were measured by western blotting. The activities of caspase-9/-3 were determined by Caspase-Glo-9/3 assay. The results indicated that Dp treatment significantly inhibited cell proliferation in a dose- and time-dependent manner, and blocked cell cycle progression at the $\mathrm{G}_{1} / \mathrm{S}$ phase in the U87MG and $\mathrm{T} 98 \mathrm{G}$ cells via the upregulation of $\mathrm{p} 53$ and $\mathrm{p} 21$ protein expression, and simultaneous downregulation of Cde25A, Cdc2 and $\mathrm{P}-\mathrm{Cdc} 2$ protein expression. Additionally, Dp treatment led to the loss of cellular mitochondrial membrane potential, and the release of cytochrome $c$, and strongly induced the occurence of apoptosis. Increased expression levels of Bim and Bax protein and the downregulated expression of $\mathrm{Bcl}-2$ protein were observed. Caspase-9/-3 were activated and their activities were elevated after Dp treatment. These findings indicate that Dp inhibits cell proliferation, induces cell cycle
\end{abstract}

Correspondence to: Dr Yongjian Dai, Department of Neurosurgery, Renmin Hospital, Hubei University of Medicine, Shiyan, Hubei 442000, P.R. China

E-mail: yongjiandai@tom.com

*Contributed equally

Abbreviations: Dp, dracorhodin perchlorate; DMSO, dimethyl sulfoxide; FBS, fetal bovine serum; PBS, phosphate-buffered saline; PI, propidium iodide; $\Delta \psi \mathrm{m}$, mitochondrial membrane potential; MTT, 3-[4,5-dimethylthiazol-2-yl]-2,5-diphenyltetrazolium bromide

Key words: dracorhodin perchlorate, apoptosis, cell cycle, glioma cells, caspase-3, caspase-9 arrest and apoptosis in glioma cells, and is a possible candidate for glioma treatment.

\section{Introduction}

Brain glioma is the most common form of neural malignancy in the nervous system. High grade glioma is the leading cause of brain cancer-related mortality due to its high invasive ability and malignant proliferation $(1,2)$. Glioma incidence accounts for approximately $40-50 \%$ of all intracranial tumors (3). Although current treatment has modestly improyed patient survival, patients with gliomas still have a very poor prognosis and low cure rates. It was reported that patients with anaplastic astrocytoma have a median survival of 2-3 years, but for those with more aggressive glioblastomas, the median survival is only $12-15$ months $(4,5)$. Currently, surgery is considered as an important initial clinical approach for glioma, yet it is difficult to completely remove diffuse tumor cells. Radiotherapy and chemotherapy after initial surgical resection are regarded as an effective therapeutic plan to prevent disease recurrence (6). However, most attempts to increase the efficacy of radiotherapy and chemotherapy are hampered by unacceptable late toxicities (7). In addition, the drug-resistance of tumor cells finally results in the abrogation of the effects of current chemotherapeutic agents (8). Accordingly, to improve the prognosis and quality of life of patients with gliomas, the identification of new anticancer agents against glioma cells requires further investigation.

Anticancer agents derived from botanical herbs such as camptothecin and paclitaxel are the drugs of first choice in many tumor therapies, accounting for more than $30 \%$ of all anticancer drugs $(9,10)$. Many scholars in China have come to believe that it is important to screen antitumor active ingredients from botanical herbs, particularly from traditional Chinese medicines.

Dracorhodin perchlorate (Dp) is a synthetic analogue of the antimicrobial anthocyanin red pigment, dracorhodin, which is isolated from the exudates of the fruit of Daemonorops draco. It is a traditional Chinese medicine (11). Increasing evidence has demonstrated that dracorhodin has various pharmacologic activities including antimicrobial and antiviral roles, but it is not stable in solution (12). Dp, one of its important derivatives, was found to inhibit cancer cell proliferation and induce apoptosis in prostate cancer PC-3 cells (13), gastric tumor SGC-7901 cells (14), breast cancer 
MCF-7 cells (15), and melanoma A375-S2 cells (16). In addition, previous studies have indicated that Dp-mediated apoptosis in human leukemia HL-60 cells was triggered through upregulation of the ratio of mitochondrial proteins, $\mathrm{Bax} / \mathrm{Bcl}-\mathrm{XL}$, and activation of caspases (17). However, according to the best of our knowledge, no literature has reported on the exact effects of Dp on glioma cells. In light of previous studies, we speculate that Dp may be an effective therapeutic candidate for glioma treatment. Therefore, in this study, we investigated the inhibitory effects of Dp on human glioma U87MG and T98G cells and the underlying molecular mechanisms. Our findings demonstrated that Dp effectively suppressed cell proliferation, arrested cell cycle progression, and induced cellular apoptosis in glioma cells via the signaling pathway of caspase-3/-9.

\section{Materials and methods}

Reagents. Dp was obtained from the National Institute for Food and Drug Control (Beijing, China), and its stock solution was prepared in dimethyl sulfoxide (DMSO) and stored at $-20^{\circ} \mathrm{C}$ for less than one month. As vehicle, $0.1 \%$ DMSO was added to the control cells, and this concentration did not show any effect on the morphology and proliferation of glioma cells. Annexin V-FITC/propidium iodide kit for apoptosis detection and Caspase-Glo-3/9 assay kit were purchased from Promega Corporation (Madison, WI, USA). ApoAlert cell fractionation kit and the JC-1 (5,5',6,6'-tetrachloro-1,1',3,3'-tetraethylbenzimidazolylcarbocyanine iodide) mitochondrial membrane potential detection kit were purchased from Cell Technology Co. (Mountain View, CA, USA). The primary antibodies against cytochrome $c$, p53, p21, Cdc2, P-Cdc2, Cdc 25A, Bim, Bax, Bcl-2, procaspase-3/-9, cleaved caspase-3/-9 and $\beta$-actin were obtained from Cell Signaling Technology (Beverly, MA, USA).

Cell lines. Human glioma cell lines U87MG and T98G were purchased from Shanghai Cell Bank of the Chinese Academy of Sciences, and they were cultured in Dulbecco's modified Eagle's medium (DMEM) supplemented with $10 \%$ fetal bovine serum (FBS) (both from Gibco, Grand Island, NY, USA) at $37^{\circ} \mathrm{C}$ in a humidified incubator with $5 \% \mathrm{CO}_{2}$.

Cell viability assay. The effect of Dp treatment on the proliferation of U87MG and T98G cells was determined by MTT assay. Briefly, the cells were seeded in 96-well plates at a density of $1 \times 10^{4}$ cells/well in a final volume of $150 \mu 1$ of culture medium with $10 \%$ FBS. After 24 h of incubation, Dp at various concentrations $(0,10,20,40,80$, and $160 \mu \mathrm{M})$ was added to each well for 24, 48 and $72 \mathrm{~h}$. Subsequently, $20 \mu \mathrm{l}$ of MTT $(5 \mathrm{mg} / \mathrm{ml}$ solution in $1 \mathrm{X}$ phosphate-buffered saline) was added to each well for a $4-\mathrm{h}$ incubation at $37^{\circ} \mathrm{C}$. The supernatant was then removed, and $150 \mu \mathrm{l}$ DMSO was added to each well. Then, cell viability was measured by an automated spectrophotometric plate reader (PerkinElmer, USA) at a wavelength of $570 \mathrm{~nm}$. The percentage of cell survival was calculated according to a previously published formula (18): Survival $(\%)=($ mean experimental absorbance $) /($ mean control absorbance) x 100. The experiments were independently performed at least three times.
Determination of cell cycle progression. The effect of Dp on cell cycle progression in the U87MG and T98G cells was carried out as described previously (19), with slight modifications. Briefly, the U87MG or T98G cells $\left(2 \times 10^{5}\right)$ were plated in 6-well plates, and treated without or with 40 and $80 \mu \mathrm{M}$ $\mathrm{Dp}$ for $48 \mathrm{~h}$. Then, the cells were trypsinized with $0.25 \%$ trypsin-EDTA solution and collected by centrifugation at 1x800 g. After washing with phosphate-buffered saline (PBS), the cells were stained with cell cycle staining solution containing propidium iodide, $1 \%$ Triton X-100, and $0.1 \mathrm{mg} / \mathrm{ml}$ ribonuclease A for $30 \mathrm{~min}$ at room temperature. Subsequently, cell cycle distribution was analyzed by FACScan (Becton Dickinson, Franklin Lakes, NJ, USA).

Determination of apoptosis. Cellular apoptosis was evaluated by using Annexin V-FITC and propidium iodide (PI) double-staining assay as described previously (20), with slight modifications. Briefly, the U87MG and T98G cells were incubated with the indicated concentrations of Dp for $48 \mathrm{~h}$, and collected for washing with PBS twice. Then, the cells were re-suspended in $500 \mu \mathrm{l}$ binding buffer $(0.14 \mathrm{M}$ $\mathrm{NaCl}, 10 \mathrm{nM}$ HEPES, and $2.5 \mathrm{mM} \mathrm{CaCl}_{2}, \mathrm{pH}$ 7.5) containing $5 \mu \mathrm{l}$ of Annexin V-FITC and $10 \mu \mathrm{l}$ of PI for $30 \mathrm{~min}$ at room temperature in the dark, followed by determination with flow cytometry (Becton Dickinson). The percentage of viable (Annexin V-FITC-negative and PI double negative), early apoptotic (Annexin V-FITC-positive and PI-negative), and late apoptotic and necrotic cells (Annexin V-FITC-positive and PI-positive) were analyzed with CellQuest software.

Measurement of mitochondrial membrane potential $(\Delta \psi m)$. JC-1, a cationic dye, was used to evaluate the extent of mitochondrial membrane potential damage as previously described (21). Briefly, U87MG and T98G cells were incubated in the absence or presence of 40 and $80 \mu \mathrm{M}$ of Dp for $24 \mathrm{~h}$, and washed with PBS (pH 7.4), followed by incubation with JC-1 staining solution at $37^{\circ} \mathrm{C}$ for $30 \mathrm{~min}$. After washing with serum-free medium, the cells were analyzed with a laser confocal scanning microscope (Olympus FY, Japan) to measure JC-1 aggregates (red) in intact mitochondria and JC-1 monomer (green) in apoptotic cells with depolarization of $\Delta \psi \mathrm{m}$. The ratio of red/green fluorescence intensity was calculated to obtain the $\Delta \psi \mathrm{m}$.

Determination of cytochrome c release. To investigate the effect of Dp on cytochrome $c$, ApoAlert cell fractionation kit was used to separate cellular cytoplasmic and mitochondrial fractions after U87MG and T98G cells were treated with 0,40 , and $80 \mu \mathrm{M}$ of Dp for $48 \mathrm{~h}$. Then, $10 \mu \mathrm{g}$ of cytosolic protein was separated on $10 \%$ SDS-PAGE gel and analyzed by western blot analysis using monoclonal anti-cytochrome $c$ and anti- $\beta$-actin antibodies as previously described (22). The densities of the cytochrome $c$ bands were normalized to the corresponding $\beta$-actin bands.

Western blotting. Human glioma U87MG and T98G cells were treated with 0,40 , and $80 \mu \mathrm{M}$ of $\mathrm{Dp}$ for $48 \mathrm{~h}$, and cell lysates were prepared as described previously (23). Subsequently, protein concentration was measured by the BCA method, and $10 \mu \mathrm{g}$ of total protein from each sample 

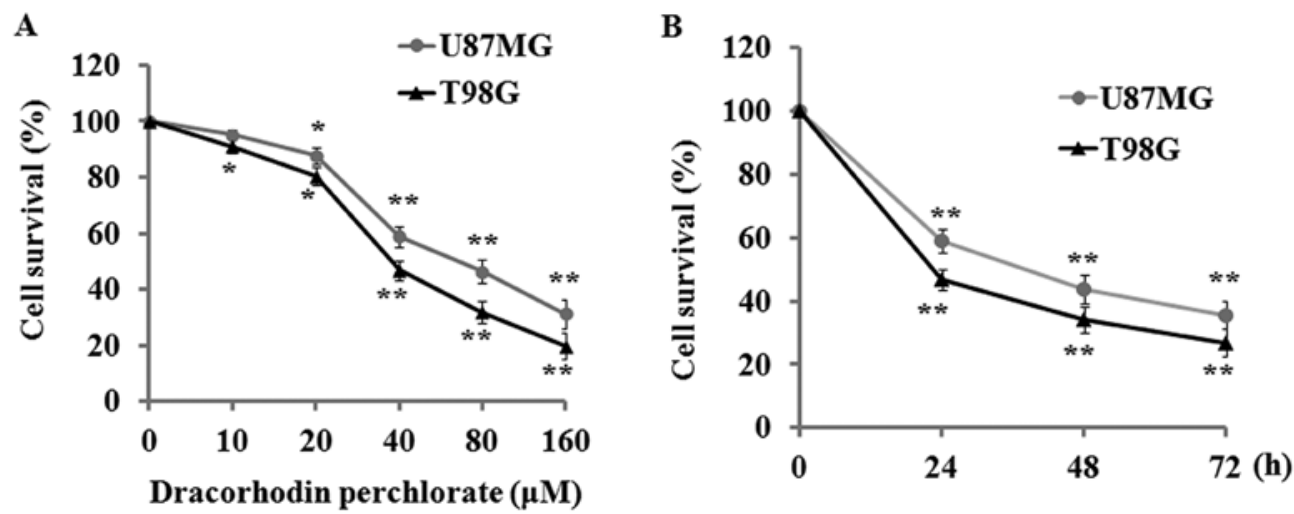

Figure 1. Effect of Dp treatment on the proliferation of human glioma U87MG and T98G cells. (A) Proliferation of U87MG and T98G cells was markedly inhibited in a dose-dependent manner after a 24 -h incubation. (B) U87MG and T98G cells were separately treated with $40 \mu \mathrm{M} \mathrm{Dp}$ for 24,48 , and $72 \mathrm{~h}$, and their growth was significantly suppressed in a time-dependent manner. The data are denoted as mean $\pm \mathrm{SD}, \mathrm{n}=3 .{ }^{*} \mathrm{P}<0.05$, and ${ }^{* * *} \mathrm{P}<0.01$, compared to the control groups.

was separated by SDS-PAGE. Then, proteins were transferred to polyvinylidene difluoride membranes, and blocked in $5 \%$ skimmed milk powder overnight at $4^{\circ} \mathrm{C}$. The membranes were washed with TBST, and separately incubated overnight at $4^{\circ} \mathrm{C}$ with the desired primary antibodies against p53, p21, Cdc2, P-Cdc2, Cdc25A, Bim, Bax, Bcl-2, procaspase-3/-9, cleaved caspase-3/-9, and $\beta$-actin used a loading control, followed by an additional washing with TBST. The membranes were then incubated with horseradish peroxidase-conjugated secondary antibodies for $1 \mathrm{~h}$ at $37^{\circ} \mathrm{C}$. Protein bands were detected using an enhanced chemiluminescence reagent and system (Amersham Biosciences Corporation, Pîscataway, NJ, USA). Band intensities were quantified by performing optical density analysis with Quantity One software (Bio-Rad, Hercules, CA, USA).

Determination of caspase-9 and caspase-3 activity. Caspase-9/-3 activity was detected as described previously (24), with slight modifications. In brief, U87MG and T98G cells were seeded in 96-well plates, and then treated with 0,40 , and $80 \mu \mathrm{M}$ of Dp for $48 \mathrm{~h}$, followed by addition of $100 \mu \mathrm{l}$ of Caspase-Glo-9 or Caspase-Glo-3 reagent to each well for $2 \mathrm{~h}$ of incubation at room temperature. Subsequently, a microplate luminometer (Promega) was used to determine the luciferase activity in the cells. Each sample was independently performed at least three times.

Statistical analysis. All data are expressed as mean \pm standard deviation (SD), and statistical analyses were performed with SPSS 13.0 software. The mean values of two groups were compared using the Student's $t$-test, and a value of $\mathrm{P}<0.05$ was considered to indicate a statistically significant result.

\section{Results}

The anti-proliferative effect of Dp on human glioma cell lines. The potential of $\mathrm{Dp}$ to suppress cell proliferation in the human glioma cell lines U87MG and T98G was first evaluated by MTT assay. The results indicated that Dp treatment for $24 \mathrm{~h}$ produced a significant dose-response inhibitory effect on U87MG and T98G cells with its corresponding concentration ranging from 10 to $160 \mu \mathrm{M}$ (Fig. 1A). Additionally, a time-dependent inhibitory effect was also found when these cell lines were incubated with $40 \mu \mathrm{M}$ Dp for 24,48 , and $72 \mathrm{~h}$ (Fig. 1B).

Dp induces cell cycle arrest in glioma cells. To investigate the mechanism involved in the suppressive effect on cell proliferation by Dp, we tested whether Dp had any effect on cell cycle progression by using flow cytometry. The results demonstrated that Dp treatment induced cell cycle arrest at the $G_{0} / G_{1}$ phase with a parallel significant reduction in the $S$ and $G_{2} / M$ phase when U87MG and T98G cells were treated with 40 and $80 \mu \mathrm{M}$ $\mathrm{Dp}$ for $48 \mathrm{~h}$ (Fig. 2A). These results suggest that cell cycle progression of U87MG and T98G cells was markedly blocked by Dp treatment.

In addition, previous studies have confirmed that tumor suppressor gene p53 plays a critical role in the regulation of its downstream genes and cell cycle progression (25). Accordingly, we further investigated p53 protein expression and cell cycle-related biomarkers including p21, Cdc25A, $\mathrm{Cdc} 2$, and $\mathrm{P}-\mathrm{Cdc} 2$. The results of western blot analysis indicated that the expression levels of p53 and p21 protein were significantly upregulated in the U87MG and T98G cells $48 \mathrm{~h}$ after Dp treatment (Fig. 2B-D). However, cell cycle-associated proteins Cdc25A, Cdc2, and P-Cdc2 were concurrently found to be downregulated in both cell lines. The findings suggest that Dp mediated cell cycle arrest possibly through modulation of the expression of p53, p21, and cell cycle-related proteins.

Dp induces apoptosis in glioma cells. To further investigate whether the decreased cell viability caused by Dp was related with apoptosis, Annexin V-FITC and propidium iodide staining was performed. As shown in Fig. 3A, Dp induced dose-dependent apoptosis when U87MG and T98G cells were exposed to various concentrations of $\mathrm{Dp}$ for $48 \mathrm{~h}$. The apoptotic percentage in the U87MG and T98G cells treated with $40 \mu \mathrm{M}$ Dp for $48 \mathrm{~h}$ was $20.93 \pm 3.42$ and $17.99 \pm 2.87 \%$, respectively. In addition, a higher percentage of apoptosis was observed when these cells were treated with $80 \mu \mathrm{M}$ Dp 
$\mathbf{A}$

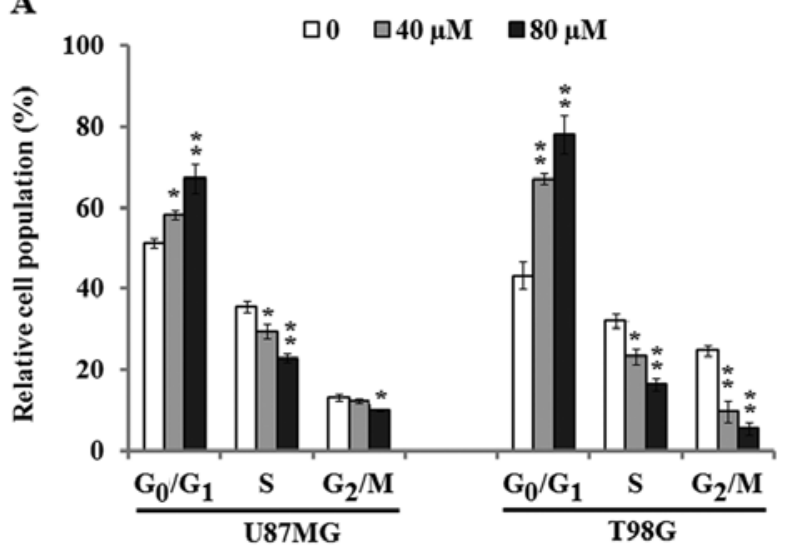

B

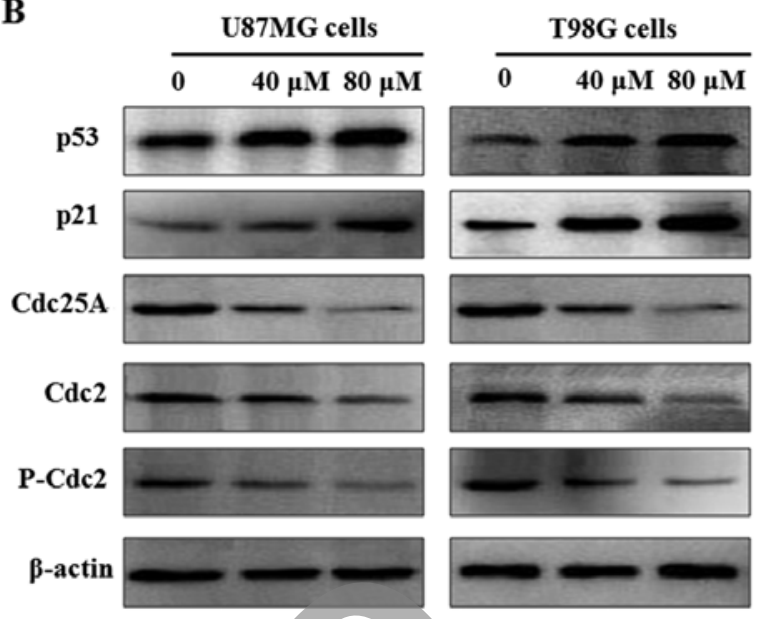

C

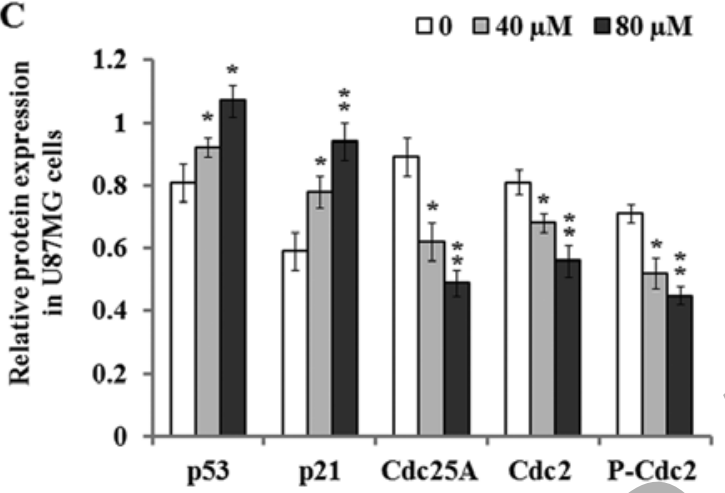

D

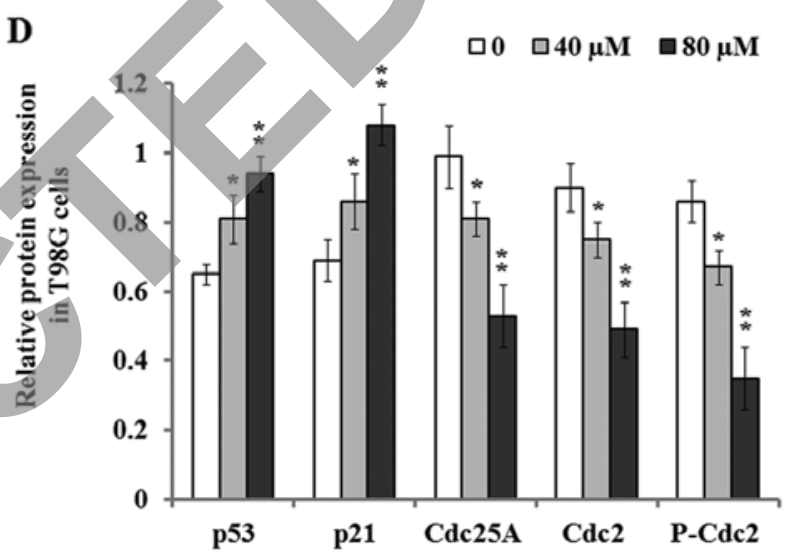

Figure 2. Effect of Dp treatment on cell cycle distribution. (A) U87MG and T98G cells were treated with 0 , 40 , and $80 \mu \mathrm{M}$ Dp for $48 \mathrm{~h}$, and the percentages of cell cycle phases were determined with flow cytometry. (B) The representative images of western blotting indicated that Dp treatment increased the expression level of p53 and p21 protein, but downregulated the expression of Cdc25A, Cdc2, and P-Cdc2 protein in the U87MG and T98G cells. (C and D) The relative expression levels of p53, p21, Cdc25A, Cdc2, and P-Cdc2 protein in U87MG (C) and T98G cells (D) were quantified by densitometry. $\beta$-actin was used as the loading control. The bars represent the mean $\pm \mathrm{SD}$ of three independent experiments. ${ }^{*} \mathrm{P}<0.05$ and ${ }^{* *} \mathrm{P}<0.01$, vs. the control.

for $48 \mathrm{~h}$. However, in the vehicle controls, the percentage of apoptosis was only $4.85 \pm 1.28$ and $5.13 \pm 1.59 \%$ in the U87MG and T98G cells (Fig. 3B).

Since mitochondrial membrane potential $(\Delta \psi \mathrm{m})$ damage occurs before apoptosis, we further investigated the change of $\Delta \psi \mathrm{m}$ by JC-1 staining that is widely used to detect mitochondrial depolarization by a decrease in the ratio of red/ green fluorescence intensity (26). The results indicated that in the Dp-treated U87MG and T98G cells, the number of cells with red staining was decreased while a concomitant increase in cells with green staining was noted, while the control cells showed strong red fluorescence and weak green fluorescence (Fig. 3C). These results demonstrated that there was a significant decrease in the ratio of red/green fluorescence intensity in the treated U87MG and T98G cells compared to the control cells (Fig. 3D), which indicated that Dp treatment resulted in the damage of $\Delta \psi \mathrm{m}$ in the U87MG and T98G cells.

$D p$ induces the release of cytochrome $c$ and activates the apoptotic pathway. To further investigate the effect of Dp on mitochondrial integrity, we measured the cytochrome $c$ release from mitochondria in the U87MG and $\mathrm{T} 98 \mathrm{G}$ cells exposed to 0,40 , and $80 \mu \mathrm{M} \mathrm{Dp}$ for $48 \mathrm{~h}$. The results indicated that the expression level of cytosolic cytochrome $c$ was enhanced with an increasing concentration of Dp in both cell lines (Fig. 4), which suggested that $\mathrm{Dp}$ treatment induced cytochrome $c$ release from the cellular mitochondria.

Additionally, we further detected the effects of Dp on apoptotic proteins Bim, Bax, and Bcl-2. The results of western blotting indicated that $\mathrm{Dp}$ treatment significantly upregulated the expression levels of pro-apoptotic proteins Bim and Bax compared to the control when U87MG and T98G cells were exposed to 40 and $80 \mu \mathrm{M}$ Dp for $48 \mathrm{~h}$. However, anti-apoptotic protein $\mathrm{Bcl}-2$ expression was found to be highly downregulated in these two cell lines, which suggested that Dp treatment suppressed Bcl-2 expression and abrogated its anti-apoptotic effect.

Dp activates caspase-9 and caspase-3 in the glioma cancer cells. Increasing studies indicate that the caspase family plays a pivotal role in induction of cellular apoptosis, in particular for the initiation and execution of apoptosis (27). Therefore, we detected the enzymatic activation of procaspase-3/-9 
A
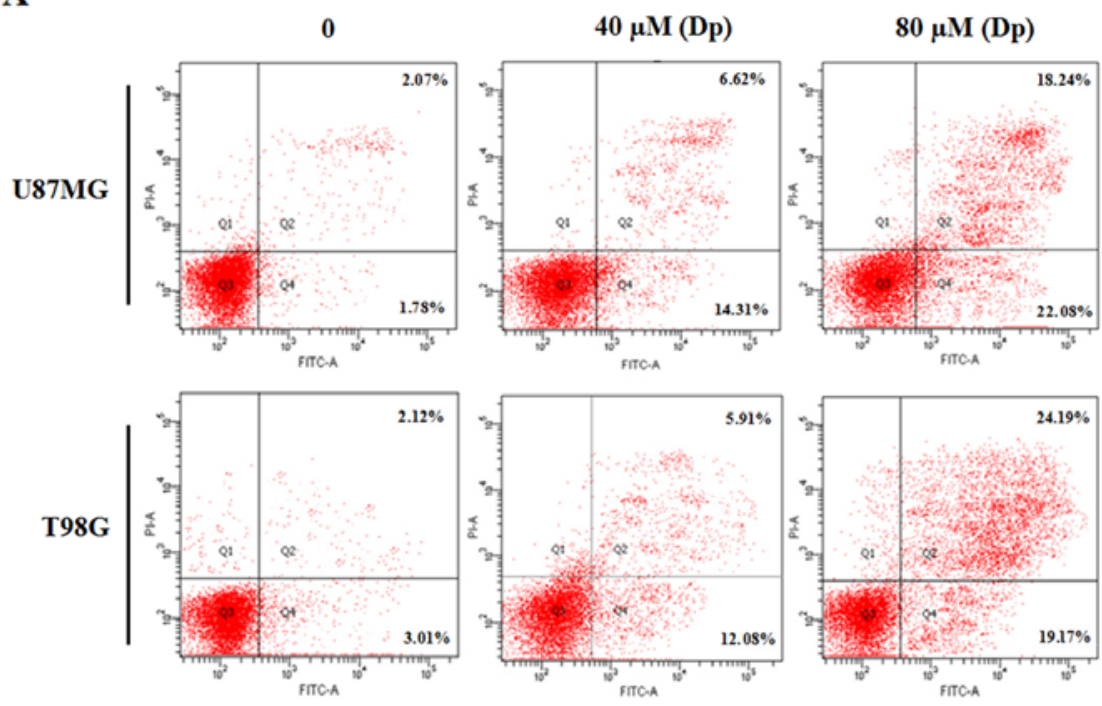

C

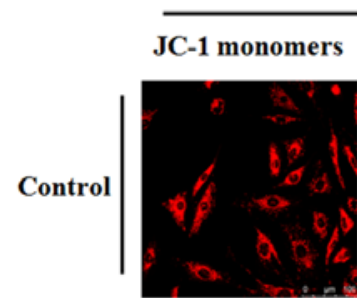

U87MG cells

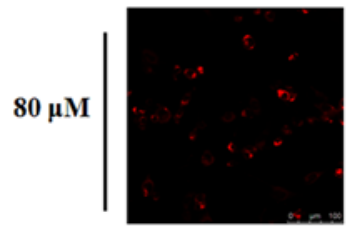

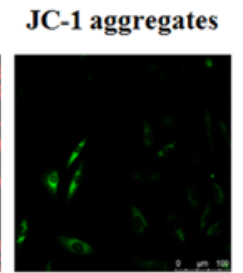

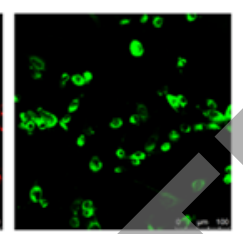

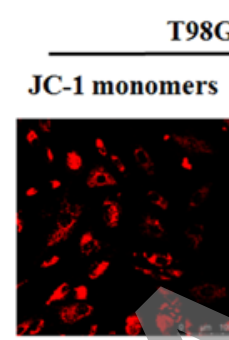

T98G cells

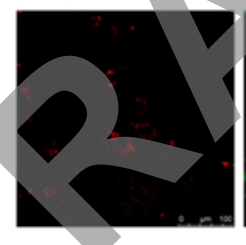

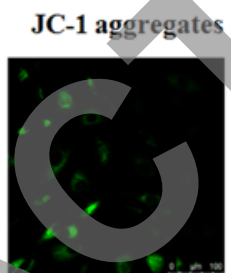

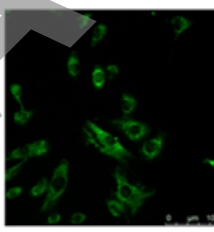

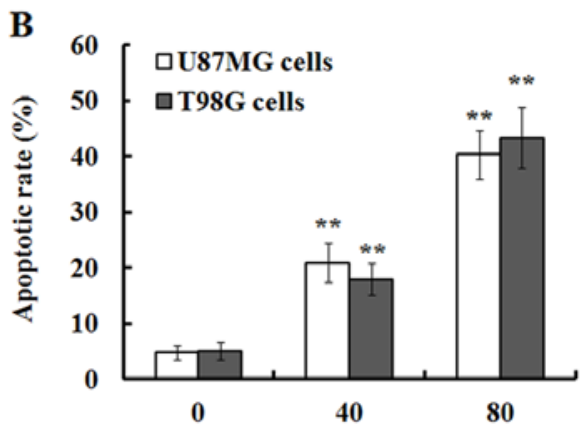

Dracorhodin perchlorate $(\mu \mathrm{M})$

B

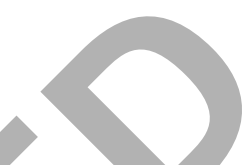

D

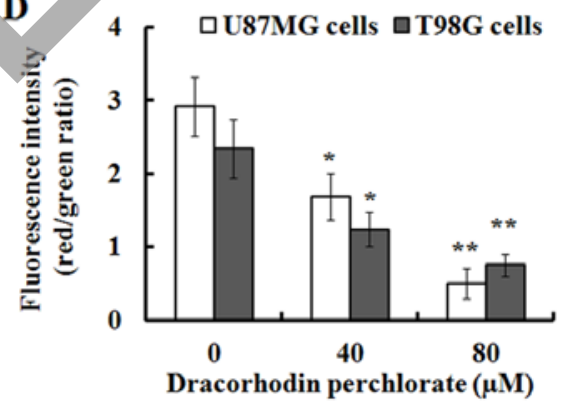

Figure 3. Dp induces apoptosis in glioma cells. (A) Cellular apoptosis was detected with Annexin V-FITC and propidium iodide (PI) double staining $48 \mathrm{~h}$ after the cells were incubated with $\mathrm{Dp}$, and the apoptotic percentages were increased in a dose-dependent manner. (B) The percentage of apoptosis in the U87MG and T98G cells from three independent experiments was quantified. (C) Mitochondrial membrane potential ( $\Delta \psi \mathrm{m})$ was monitored under a laser confocal scanning microscope with fluorescent dye JC- 1 staining, and the representative micrographs of $\Delta \psi \mathrm{m}$ change can be observed in the U87MG and T98G cells (D) The change in $\Delta \psi \mathrm{m}$ was quantified by the ratio of red/green fluorescence intensity in the U87MG and T98G cells. The data are denoted as mean \pm SD of three independent experiments. $\mathrm{P}<0.05$ and ${ }^{*} \mathrm{P}<0.01$, compared with the control groups.

during Dp-mediated apoptosis in the U87MG and T98G cells. The results of the western blot analysis demonstrated that a gradual reduction of intact procaspase-3/-9 was observed $48 \mathrm{~h}$ after the cells were treated with 0,40 , and $80 \mu \mathrm{M}$ Dp, while their proteolytic cleaved forms, caspase-3/-9, were synchronously found to be upregulated (Fig. 5A). Further quantification analysis indicated that the expression of procaspase-3/-9 protein was significantly decreased, while cleaved caspase-3/-9 was significantly increased when compared to the controls (Fig. 5B and C). These results suggest that Dp treatment induced the activation of caspase-3/-9, which was further confirmed by their activity analysis. As shown in Fig. 5D and E, the results of the Caspase-Glo-3/9 assays demonstrated that their activity was significantly enhanced in the Dp-treated U87MG and T98G cells in a concentrationdependent manner compared to the control. These findings suggest that Dp induced cellular apoptosis in U87MG and T98G cells probably via the caspase-dependent pathway.

\section{Discussion}

Malignant gliomas are the most common and fatal primary tumors of the central nervous system, and account for approximately $70 \%$ of the 22,500 new cases diagnosed in the United States each year $(28,29)$. Even when a combination of surgery, radiotherapy and chemotherapy is applied, the outlook for patients with malignant gliomas remains very poor. Resistance to apoptosis is a characteristic of many types of cancer including malignant glioma, and finally abrogates the effects of radiotherapy and chemotherapy (18). Therefore, development of novel therapeutic strategies and discovery of new drugs are essential to improve the prognosis of patients with malignant gliomas (29). In the present study, we investigated Dp for its capability to suppress cell proliferation in human glioma cells. Our results indicated that Dp had strong inhibitory effects on glioma U87MG and T98G cells through cell cycle arrest at the $\mathrm{G}_{1}$ phase and induction of apoptosis. 
A

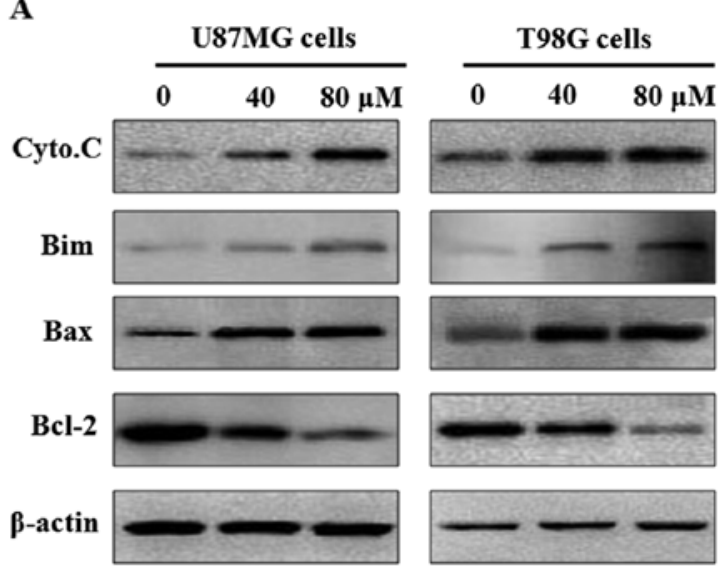

B

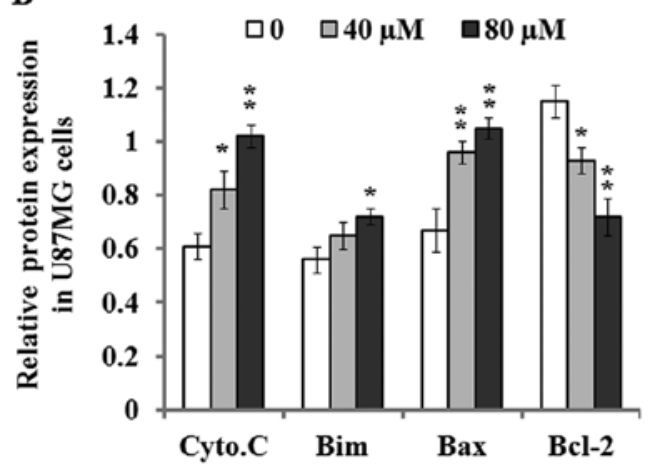

Figure 4. The effects of Dp on cytochrome $c$ release and Bim, Bax, and Bcl-2 expression. Cell lysates or cytosolic extracts were respectively prepared as described in Materials and methods after the cells were exposed to 0, 40, and $80 \mu \mathrm{M}$ of Dp for $48 \mathrm{~h}$, and subjected to $10 \%$ SDS-PAGE for western blot analysis. (A) The representative images of western blot analysis indicated that Dp treatment resulted in the increase of cytochrome $c$ release, and upregulated the expression levels of Bim and Bax proteins, and concurrently decreased Bcl-2 protein expression in the U87MG and T98G cells. $\beta$-actin was used as the loading control. (B and C) The protein expression levels of cytochrome $c$, Bim, Bax, and Bcl-2 in U87MG (B) and T98G (C) cells were quantified by densitometric analysis of targeted bands against $\beta$-actin bands. Each independent experiment was performed 3 times. ${ }^{*} \mathrm{P}<0.05$ and ${ }^{* *} \mathrm{P}<0.01$, vs. the control. Cyto.C, cytochrome $c$.

Dracorhodin is a major constituent found in the resin of Daemonorops draco and is a traditional Chinese medicine for wound healing, pain alleviation, and bleeding control (30). Recently, dracorhodin and its derivant, Dp, were found to have similar pharmacologic activities, and many researchers have focused on their potential antitumor activity (12). Previous studies found that Dp inhibits cell growth and induces apoptosis in various cancer cells (14-17). In this study, we firstly found that Dp effectively suppressed the proliferation of human glioma U87MG and T98G cells. Furthermore, we also found that the growth inhibitory effect of Dp on glioma cells was associated with arrest of the cell cycle and induction of apoptosis.

Previous studies have demonstrated that Dp inhibited cell proliferation in various cancer cell lines, yet the effects of Dp treatment on cell cycle progression have not been evaluated. In this study, we confirmed that Dp treatment resulted in cell cycle arrest at the $\mathrm{G}_{1}$ phase in U87MG and T98G cells, concurrently reducing the percentage of cells at the $\mathrm{S}$ and $\mathrm{G}_{2} / \mathrm{M}$ phase (Fig. 2). To further investigate the mechanism of Dp-mediated cell cycle arrest in glioma cells, we detected the expression levels of cell cycle-associated proteins such as p53, p21, Cdc25A, Cdc2 and its phosphorylated form. The results of western blot analysis indicated that the expression levels of p53 and p21 proteins were significantly upregulated by Dp treatment. It is well-known that p53 functions as a focal point for determining whether cells respond to various types and levels of stress and treatment with cell cycle arrest, DNA repair, autophagy, cell metabolism, senescence and apoptosis (31). Previous studies have indicated that $\mathrm{p} 21$ is one of the most important downstream genes of p53, and contributes to the regulation of cell cycle progression at the $\mathrm{G}_{1} / \mathrm{S}$ phase (32), and recruitment of co-activators including Tip 60/hMOF and CBP/p300 is essential for the activation of p21 in a p53-dependent manner $(33,34)$. Therefore, in the present study, we speculated that cell cycle arrest at $G_{1}$ phase in glioma cells after $\mathrm{Dp}$ treatment was mediated by the regulation of p53 and p21 protein expression. However, whether Dp treatment results in the translocation of p21 in the subcellular location was not investigated, and needs to be clarified in future research. Previous studies indicate that Cdc25A and $\mathrm{Cdc} 2$ play an important role in cell cycle progression at the $\mathrm{G}_{1} / \mathrm{S}$ or $\mathrm{G}_{2} / \mathrm{M}$ checkpoint $(35,36)$. Similar to previous studies, the downregulation of $\mathrm{Cdc} 25 \mathrm{~A}, \mathrm{Cdc} 2$ and P-Cdc2 expression at least contributed to the inhibition of cell cycle progression in the U87MG and T98G cells. 
A

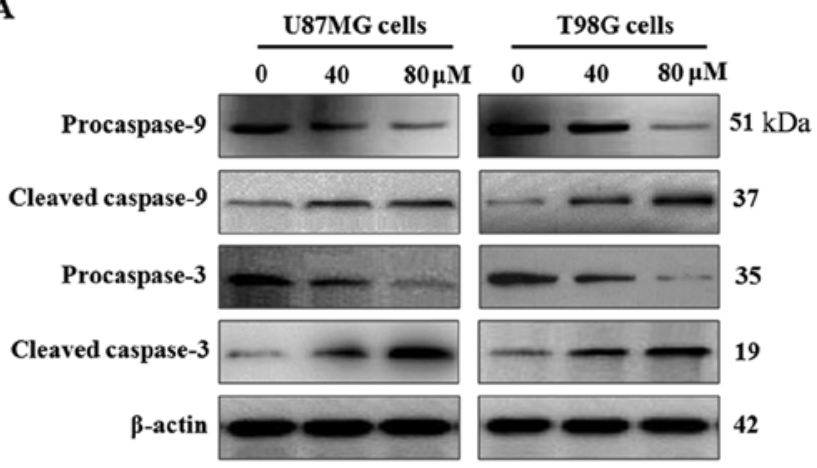

B

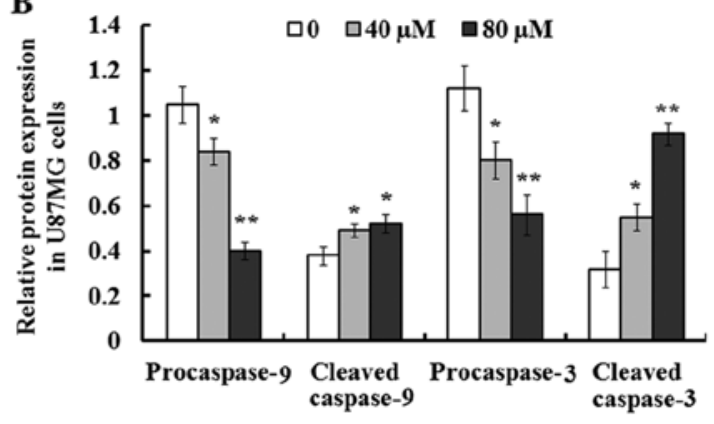

C

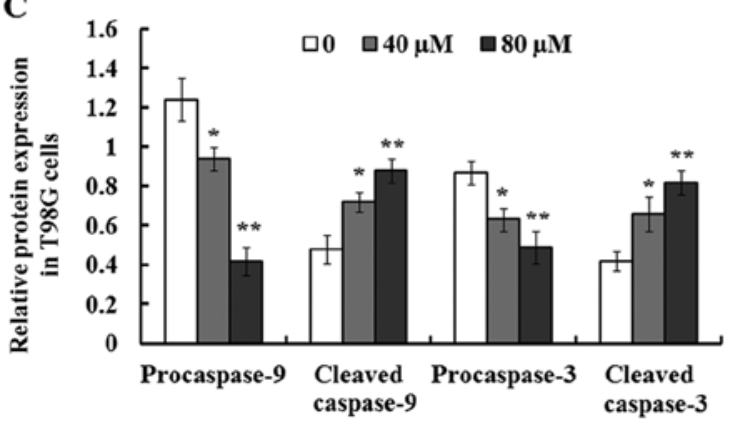

D

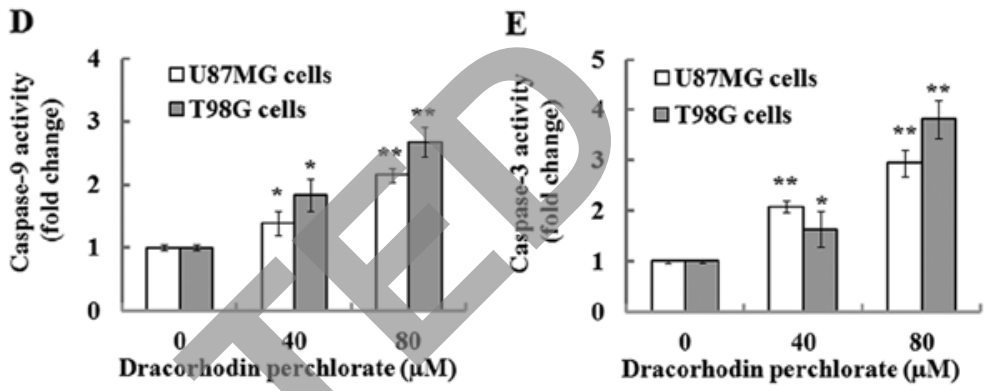

Figure 5. The effects of Dp on the expression and activity of caspase-3/-9. (A) Representative western blot images for detecting the protein expression levels of procaspase-3/-9 and cleaved caspase-3/-9 in U87MG and T98G cells $48 \mathrm{~h}$ after Dp treatment. (B and C) The relative expression levels of procaspase-3/-9 and cleaved caspase-3/-9 proteins in U87MG (B) and T98G (C) cells were quantified by densitometry from three independent experiments. (D and E) The activity of caspase-9 (D) and caspase-3 (E) in U87MG and T98G cells was respectively determined $48 \mathrm{~h}$ after incubation with 0,40 , and $80 \mu \mathrm{M}$ of Dp. "P<0.05 and ${ }^{* * *} \mathrm{P}<0.01$, in contrast to the control groups.

The induction of apoptosis is regarded as an effective approach for cancer control and therapy. Accordingly, we investigated whether Dp-mediated anti-proliferation effects in glioma cells was due to the occurrence of apoptosis apart from cell cycle arrest. Our results indicated that a significant increase in the percentage of apoptosis in U87MG and T98G cells was observed after Dp treatment. Further investigation demonstrated that the damage of mitochondrial membrane potential and the following release of cytochrome $c$ from the mitochondria confirmed the occurrence of apoptosis in these cells, similar to a previous study (15). Research has confirmed that $\mathrm{p} 53$ is an important regulator of apoptosis, and its interaction with Bcl-2 or Bcl-XL results in the assembly of pro-apoptotic proteins Bax and Bak in the mitochondrial membrane to form pores, which finally causes the release of cytochrome $c$ and other apoptotic activators from the mitochondria (37). It was reported that the mitochondrial pathway (also called the intrinsic pathway) of apoptosis is inhibited or triggered by anti-apoptotic proteins such as Bcl-2 and Bcl-xL or pro-apoptotic proteins such as Bax, Bak, Bim and Bid (38). In the present study, the downregulation of $\mathrm{Bcl}-2$ expression, and the synchronously upregulated expression of Bim and Bax in the U87MG and T87G cells probably contributed to the release of cytochrome $c$ and the following apoptotic events, which is similar to previous studies in human gastric tumor cells and breast cancer cells $(14,15)$.

It is well-known that the caspase family plays a critical role in apoptotic initiation and execution (39). In the present study, our findings showed that Dp treatment resulted in the downregulation of procaspase- 9 and procaspase- 3 proteins in the U87MG and T98G cells, and concurrently upregulated the expression levels of cleaved caspase- 9 and caspase-3, which indicated that procaspase- 9 and procaspase- 3 were degraded by proteolytic cleavage, and their corresponding cleaved fragments (cleaved caspase-9/-3) could be detected by western blotting. These findings indicated that caspase- 9 and caspase- 3 were activated by Dp treatment during apoptosis in the U87MG and T98G cells. Caspase-Glo-3/9 assays also confirmed that their activities were both significantly increased in these cells, which were indispensable for their roles in apoptosis. Previous studies also demonstrated that $\mathrm{Dp}$ treatment led to apoptosis in gastric cancer SGC-7901 cells (14), breast cancer MCF-7 cells (15), and leukemia HL-60 cells (17) via degradation of caspase-1, $-3,-8$, and -9 , and increased their activities. Additionally, caspase- 9 activation by a complex containing Apaf-1 and cytochrome $c$ can trigger the activation of other caspase family members including caspase- $3,-6$, and -7 , finally resulting in the occurrence of apoptosis $(40,41)$.

Taken together, our findings indicated that Dp treatment effectively inhibited cell proliferation and arrested the cell cycle at $\mathrm{G}_{1}$ phase, and concurrently decreased the percentage of cells at the $S$ and $G_{2}$ phase in glioma U87MG and T98G cells. The arrest of the cell cycle was regulated through the mediation of the cell cycle regulatory proteins including the upregulation of p53 and p21 protein production, and synchronous reduction in the protein expression levels of Cdc25A, $\mathrm{Cdc} 2$ and its phosphorylated form. In addition, we found that Dp treatment induced cellular apoptosis in the U87MG and T98G cells in a concentration-dependent manner, and resulted in mitochondrial membrane potential loss and the release of 
cytochrome $c$. Moreover, further investigation indicated that the expression levels of pro-apoptotic Bim and Bax proteins were enhanced, while the level of anti-apoptotic Bcl-2 protein was simultaneously decreased by Dp treatment, resulting in the activation of caspase- 9 and caspase-3, and finally inducing cellular apoptosis in the U87MG and T98G cells probably via the mitochondrial pathway. Therefore, our data suggest that $\mathrm{Dp}$ is possibly a potential candidate for glioma treatment.

\section{Acknowledgements}

We would like to thank Dr Martin Nolan for the English language editing of this manuscript.

\section{References}

1. Guo X, Fan W, Bian X and Ma D: Upregulation of the Kank1 gene-induced brain glioma apoptosis and blockade of the cell cycle in G0/G1 phase. Int J Oncol 44: 797-804, 2014.

2. Li Q, Shen K, Zhao Y, Ma C, Liu J and Ma J: miR-92b inhibitor promoted glioma cell apoptosis via targeting DKK3 and blocking the Wnt/beta-catenin signaling pathway. J Transl Med 11: 302, 2013.

3. Xu C, Sun G, Yuan G, Wang R and Sun X: Effects of platycodin D on proliferation, apoptosis and PI3K/Akt signal pathway of human glioma U251 cells. Molecules 19: 21411-21423, 2014.

4. Sun T, Zhang Z, Li B, Chen G, Xie X, Wei Y, Wu J, Zhou Y and $\mathrm{Du} \mathrm{Z}$ : Boron neutron capture therapy induces cell cycle arrest and cell apoptosis of glioma stem/progenitor cells in vitro. Radiat Oncol 8: 195, 2013.

5. Shang C, Hong Y, Guo Y, Liu YH and Xue YX: miR-210 up-regulation inhibits proliferation and induces apoptosis in glioma cells by targeting SIN3A. Med Sci Monit 20: 2571-2577, 2014.

6. Knizhnik AV, Roos WP, Nikolova T, Quiros S, Tomaszowski KH, Christmann M and Kaina B: Survival and death strategies in glioma cells: Autophagy, senescence and apoptosis triggered by a single type of temozolomide-induced DNA damage. PLoS One 8: e55665, 2013.

7. Sharma A, Sharma DN, Julka PK and Rath GK: Treatment options in elderly patients with glioblastoma. Lancet Oncol 13: e460-e461, author reply e461-e462, 2012

8. Mhaidat NM, Bouklihacene M and Thorne RF: 5-Fluorouracilinduced apoptosisin colorectalcancercellsis caspase-9-dependent and mediated by activation of protein kinase $C-\delta$. Oncol Lett 8 : 699-704, 2014.

9. Auyeung KK, Cho $\mathrm{CH}$ and $\mathrm{Ko}$ JK: A novel anticancer effect of Astragalus saponins: Transcriptional activation of NSAID-activated gene. Int J Cancer 125: 1082-1091, 2009.

10. Ando M, Yonemori K, Katsumata N, Shimizu C, Hirata T, Yamamoto $\mathrm{H}$, Hashimoto $\mathrm{K}$, Yunokawa M, Tamura $\mathrm{K}$ and Fujiwara Y: Phase I and pharmacokinetic study of nab-paclitaxel, nanoparticle albumin-bound paclitaxel, administered weekly to Japanese patients with solid tumors and metastatic breast cancer. Cancer Chemother Pharmacol 69: 457-465, 2012.

11. Rao GS, Gerhart MA, Lee RT III, Mitscher LA and Drake S: Antimicrobial agents from higher plants. Dragon's blood resin. J Nat Prod 45: 646-648, 1982.

12. Zhang P, Li J, Tang X, Zhang J, Liang J and Zeng G: Dracorhodin perchlorate induces apoptosis in primary fibroblasts from human skin hypertrophic scars via participation of caspase-3. Eur J Pharmacol 728: 82-92, 2014.

13. He Y, Ju W, Hao H, Liu Q, Lv L and Zeng F: Dracorhodin perchlorate suppresses proliferation and induces apoptosis in human prostate cancer cell line PC-3. J Huazhong Univ Sci Technolog Med Sci 31: 215-219, 2011.

14. Rasul A, Ding C, Li X, Khan M, Yi F, Ali M and Ma T: Dracorhodin perchlorate inhibits PI3K/Akt and $\mathrm{NF}-\kappa \mathrm{B}$ activation, up-regulates the expression of $\mathrm{p} 53$, and enhances apoptosis. Apoptosis 17: 1104-1119, 2012.

15. Yu JH, Zheng GB, Liu CY, Zhang LY, Gao HM, Zhang YH, Dai CY, Huang L, Meng XY, Zhang WY, et al: Dracorhodin perchlorate induced human breast cancer MCF-7 apoptosis through mitochondrial pathways. Int J Med Sci 10: 1149-1156, 2013.
16. Xia M, Wang M, Tashiro S, Onodera S, Minami M and Ikejima T: Dracorhodin perchlorate induces A375-S2 cell apoptosis via accumulation of $\mathrm{p} 53$ and activation of caspases. Biol Pharm Bull 28: 226-232, 2005.

17. Xia MY, Wang MW, Cui Z, Tashiro SI, Onodera S, Minami M and Ikejima T: Dracorhodin perchlorate induces apoptosis in HL-60 cells. J Asian Nat Prod Res 8: 335-343, 2006.

18. Yan YY, Bai JP, Xie Y, Yu JZ and Ma CG: The triterpenoid pristimerin induces U87 glioma cell apoptosis through reactive oxygen species-mediated mitochondrial dysfunction. Oncol Lett 5: 242-248, 2013.

19. Bai Y, Mao QQ, Qin J, Zheng XY, Wang YB, Yang K, Shen HF and Xie LP: Resveratrol induces apoptosis and cell cycle arrest of human T24 bladder cancer cells in vitro and inhibits tumor growth in vivo. Cancer Sci 101: 488-493, 2010.

20. Wu H, Jiang H, Lu D, Xiong Y, Qu C, Zhou D, Mahmood A and Chopp M: Effect of simvastatin on glioma cell proliferation, migration, and apoptosis. Neurosurgery 65: 1087-1096, discussion 1096-1097, 2009.

21. Selvaraj V, Armistead MY, Cohenford M and Murray E: Arsenic trioxide $\left(\mathrm{As}_{2} \mathrm{O}_{3}\right)$ induces apoptosis and necrosis mediated cell death through mitochondrial membrane potential damage and elevated production of reactive oxygen species in PLHC-1 fish cell line. Chemosphere 90: 1201-1209, 2013.

22. Zhou L, Guo X, Chen M, Fu S, Zhou J, Ren G, Yang Z and Fan W: Inhibition of $\delta$-opioid receptors induces brain glioma cell apoptosis through the mitochondrial and protein kinase $\mathrm{C}$ pathways. Oncol Lett 6: 1351-1357, 2013.

23. Li ZH, Yu Y, DU C, Fu H, Wang J and Tian Y: RNA interference-mediated USP22 gene silencing promotes human brain glioma apoptosis and induces cell cycle arrest. Oncol Lett 5: 1290-1294, 2013

24. Malekinejad H, Moradi M and Fink-Gremmels J: Cytochrome C and caspase-3/7 are involved in mycophenolic acid-induced apoptosis in genetically engineered PC12 neuronal cells expressing the p53 gene. Iran J Pharm Res 13: 191-198, 2014.

25. Kheirollahi M, Mehr-Azin M, Kamalian N and Mehdipour P: Expression of cyclin D2, P53, Rb and ATM cell cycle genes in brain tumors. Med Oncol 28: 7-14, 2011

26. Shieh JM, Huang TF, Hung CF, Chou KH, Tsai YJ and Wu WB: Activation of c-Jun N-terminal kinase is essential for mitochondrial membrane potential change and apoptosis induced by doxycycline in melanoma cells. Br J Pharmacol 160: 1171-1184, 2010.

27. McStay GP and Green DR: Measuring apoptosis: Caspase inhibitors and activity assays. Cold Spring Harb Protoc 2014: 799-806, 2014.

28. Wen PY and Kesari S: Malignant gliomas in adults. N Engl J Med 359: 492-507, 2008.

29. Liu WT, Huang CY, Lu IC and Gean PW: Inhibition of glioma growth by minocycline is mediated through endoplasmic reticulum stress-induced apoptosis and autophagic cell death. Neuro Oncol 15: 1127-1141, 2013.

30. Shi J, Hu R, Lu Y, Sun C and Wu T: Single-step purification of dracorhodin from dragon's blood resin of Daemonorops draco using high-speed counter-current chromatography combined with pH modulation. J Sep Sci 32: 4040-4047, 2009.

31. Kruse JP and Gu W: Modes of p53 regulation. Cell 137: 609-622, 2009.

32. Armstrong MJ, Stang MT, Liu Y, Gao J, Ren B, Zuckerbraun BS, Mahidhara RS, Xing Q, Pizzoferrato E and Yim JH: Interferon regulatory factor 1 (IRF-1) induces p21(WAF1/CIP1) dependent cell cycle arrest and p21(WAF1/CIP1) independent modulation of survivin in cancer cells. Cancer Lett 319: 56-65, 2012.

33. Sykes SM, Mellert HS, Holbert MA, Li K, Marmorstein R, Lane WS and McMahon SB: Acetylation of the p53 DNA-binding domain regulates apoptosis induction. Mol Cell 24: 841-851, 2006.

34. Tang Y, Luo J, Zhang W and Gu W: Tip60-dependent acetylation of p53 modulates the decision between cell-cycle arrest and apoptosis. Mol Cell 24: 827-839, 2006.

35. Tu YS, Kang XL, Zhou JG, Lv XF, Tang YB and Guan YY: Involvement of Chk1-Cdc25A-cyclin A/CDK2 pathway in simvastatin induced S-phase cell cycle arrest and apoptosis in multiple myeloma cells. Eur J Pharmacol 670: 356-364, 2011.

36. Watanabe G, Behrns KE, Kim JS and Kim RD: Heat shock protein 90 inhibition abrogates hepatocellular cancer growth through cdc2-mediated G2/M cell cycle arrest and apoptosis. Cancer Chemother Pharmacol 64: 433-443, 2009. 
37. Tomita Y, Marchenko N, Erster S, Nemajerova A, Dehner A, Klein C, Pan H, Kessler H, Pancoska P and Moll UM: WT p53, but not tumor-derived mutants, bind to $\mathrm{Bcl} 2$ via the DNA binding domain and induce mitochondrial permeabilization. J Bio Chem 281: 8600-8606, 2006.

38. Kassi E, Sourlingas TG, Spiliotaki M, Papoutsi Z, Pratsinis H, Aligiannis $\mathrm{N}$ and Moutsatsou P: Ursolic acid triggers apoptosis and Bcl-2 downregulation in MCF-7 breast cancer cells. Cancer Invest 27: 723-733, 2009.

39. Das S, Dey KK, Bharti R, MaitiChoudhury S, Maiti S and Mandal M: PKI 166 induced redox signalling and apoptosis through activation of p53, MAP kinase and caspase pathway in epidermoid carcinoma. J Exp Ther Oncol 10: 139-153, 2012.
40. Breckenridge DG and Xue D: Regulation of mitochondrial membrane permeabilization by BCL-2 family proteins and caspases. Curr Opin Cell Biol 16: 647-652, 2004.

41. Feng R, Han J, Ziegler J, Yang M and Castranova V: Apaf-1 deficiency confers resistance to ultraviolet-induced apoptosis in mouse embryonic fibroblasts by disrupting reactive oxygen species amplification production and mitochondrial pathway. Free Radic Biol Med 52: 889-897, 2012. 